VoL. 48 (1993) [265-274]

\title{
THE HOMOLOGICAL DIMENSIONS OF SIMPLE MODULES
}

\author{
NanqINg Ding and Jianlong Chen
}

\begin{abstract}
We prove that (a) if $R$ is a commutative coherent ring, the weak global dimension of $R$ equals the supremum of the flat (or (FP-) injective) dimensions of the simple $R$-modules; (b) if $R$ is right semi-artinian, the weak (respectively, the right) global dimension of $R$ equals the supremum of the flat (respectively, projective) dimensions of the simple right $R$-modules; (c) if $R$ is right semi-artinian and right coherent, the weak global dimension of $R$ equals the supremum of the $F P$-injective dimensions of the simple right $R$-modules.
\end{abstract}

\section{INTRODUCTION}

In this paper $R$ will denote an associative ring with identity and all modules will be unitary. Following [12], the projective (respectively, injective, flat) dimension of an $R$-module $M$ will be denoted by $p d M$ (respectively, $i d M, f d M$ ), and the left (respectively, the right, the weak) global dimension of $R$ will be denoted by $\ell D(R)$ (respectively $r D(R), w D(R)$ ).

It is well known that $\ell D(R)$ is computed by Auslander's classical formula [2] as

$$
\ell D(R)=\sup \{p d M \mid M \text { is a cyclic left } R \text {-module }\}
$$

In general, there is no analogy to Auslander's formula in terms of injective dimensions of cyclic modules, although if $R$ is left Noetherian we do get one [10]. For special classes of rings $R$ the number of cyclics to be checked in computing the (weak) global dimension of $R$ may be reduced. For example if $R$ is a commutative Noetherian ring or a right coherent and left $F B N$ ring, then it is sufficient to check the projective (or injective) dimensions of simple modules $[11,17]$. The purpose of this paper is to prove that if $R$ is a commutative coherent ring or a right semi-artinian ring, then we may compute the (weak) global dimension of $R$ using just the homological dimensions of simple modules. The main results are as follows.

I. Let $R$ be a commutative coherent ring. Then

(a) $\quad p d A=\sup \left\{n \mid \operatorname{Ext}^{n}(A, S) \neq 0\right.$ for some simple $R$-module $\left.S\right\}$ $=\sup \left\{n \mid \operatorname{Tor}_{n}(A, S) \neq 0\right.$ for some simple $R$-module $\left.S\right\}$

for any finitely presented $R$-module $A$.

Received 6 October 1992

Copyright Clearance Centre, Inc. Serial-fee code: 0004-9729/93 \$A2.00+0.00. 
(b) $w D(R)=\sup \{f d S \mid S$ is a simple $R$-module $\}$ $=\sup \{i d S \mid S$ is a simple $R$-module $\}$ $=\sup \{F P$-idS $\mid S$ is a simple $R$-module $\}$.

II. If $R$ is a right semi-artinian ring, then

(a) $f d A=\sup \left\{n \mid \operatorname{Tor}_{n}(S, A) \neq 0\right.$ for some simple right $R$-module $\left.S\right\}$ for any left $R$-module $A$.

(b) $i d A=\sup \left\{n \mid \operatorname{Ext}^{n}(S, A) \neq 0\right.$ for some simple right $R$-module $\left.S\right\}$ for any right $R$-module $A$.

(c) $w D(R)=\sup \{f d S \mid S$ is a simple right $R$-module $\}$.

(d) $r D(R)=\sup \{p d S \mid S$ is a simple right $R$-module $\}$.

III. Let $R$ be a right semi-artinian and right coherent ring. Then

(a) $p d A=\sup \left\{n \mid \operatorname{Ext}^{n}(A, S) \neq 0\right.$ for some simple right $R$-module $\left.S\right\}$ for any finitely presented right $R$-module $A$.

(b) $w D(R)=\sup \{F P$-idS $\mid S$ is a simple right $R$-module $\}$.

For all $R$-modules $M, N$, $\operatorname{Hom}(M, N)$ will mean $\operatorname{Hom}_{R}(M, N)$, and similarly $M \otimes N$ will denote $M \otimes_{R} N$ unless otherwise specified.

\section{Preliminaries}

In this section, we shall recall several known notions which we need in the later sections.

(1) An $R$-module $M$ is called $F P$-injective if $\operatorname{Ext}^{1}(N, M)=0$ for all finitely presented modules $N$. The $F P$-injective dimension of $M$, denoted by $F P$-idM, is defined to be the least nonnegative integer $n$ such that $\operatorname{Ext}^{n+1}(N, M)=0$ for all finitely presented modules $N$. If no such $n$ exists, set $F P$-idM $=\infty[15,6]$.

(2) A ring is called a right coherent ring if every finitely generated right ideal of $R$ is finitely presented. For details see $[\mathbf{3}, \mathbf{8}, \mathbf{1 5}]$.

(3) A right $R$-module $M$ is called semi-artinian if every non-zero quotient module of $M$ has non-zero socle. A ring $R$ is said to be right semiartinian if it is semi-artinian as a right $R$-module. By [16, Proposition 2.5], $R$ is right semi-artinian if and only if every right $R$-module is semiartinian. A ring $R$ is called a right $S F$-ring if all simple right $R$-modules are flat [4].

(4) Let $\mathfrak{A}$ be a nonempty collection of right ideals of a ring $R$. Following [14], a right $R$-module $X$ is said to be $\mathfrak{A}$-injective provided each $R$-homomorphism $f: A \rightarrow X$ with $A$ in $\mathfrak{A}$ can be extended to an $R$ homomorphism $g: R \rightarrow X$. 


\section{SIMPLE MOdULES OVER COMMUTATIVE COHERENT RINGS}

The proof of the main theorem of this section depends on the following lemmas.

Lемма 3.1. Let $R$ be a commutative ring, $M$ an $R$-module and $S$ a simple $R$-module. Then

(1) $\operatorname{Tor}_{n}(M, S)=0$ if and only if $\operatorname{Ext}^{n}(M, S)=0$ for an integer $n \geqslant 0$.

(2) $f d S=i d S=F P$-idS.

Proof: It is easy to see that (1) implies (2). We now prove (1). Let $E$ be the injective envelope of the direct sum of one copy of each of the simple $R$-modules. Thus $E=E\left(\bigoplus_{i \in I} S_{i}\right)$ where $\left\{S_{i}\right\}_{i \in I}$ is the family of all (isomorphism types) of simple $R$ modules and if $i \neq j$ then $S_{i} \not S_{j}$. Then $E$ is an injective cogenerator by $[1$, Corollary 18.19] and $\operatorname{Hom}(S, E) \cong S$ as $R$-modules by the proof of [18, Lemma 2.6]. Since $E$ is injective, we have an isomorphism

$$
\operatorname{Ext}^{n}(M, \operatorname{Hom}(S, E)) \cong \operatorname{Hom}\left(\operatorname{Tor}_{n}(M, S), E\right)
$$

that is $\operatorname{Ext}^{n}(M, S) \cong \operatorname{Hom}\left(\operatorname{Tor}_{n}(M, S), E\right)$. Therefore

$$
\operatorname{Tor}_{n}(M, S)=0 \text { if and only if } \operatorname{Ext}^{n}(M, S)=0
$$

since $E$ is a cogenerator.

LEMMA 3.2. Let $R$ be a commutative coherent local ring with only one maximal ideal $m$ and $M$ a finitely presented $R$-module. Then

$$
p d M \leqslant n \text { if and only if } \operatorname{Tor}_{n+1}(M, R / m)=0 \text {. }
$$

Proof: See Rotman [12, Lemma 9.53]. His argument remains valid in our setting.

LEMMA 3.3. Let $R$ be a commutative ring and $A$ an $R$-module, then

$$
f d A=\sup \left\{f d_{R_{m}} A_{m} \mid m \text { is a maximal ideal of } R\right\}
$$

Proof: Clear.

LEмMA 3.4. Let $R$ be a commutative coherent ring and $A$ a finitely presented $R$-module. Then the following are equivalent:

(1) $p d A \leqslant n$.

(2) $\operatorname{Tor}_{n+1}(A, S)=0$ for all simple $R$-modules $S$. 
Proof: $(1) \Rightarrow(2)$ is trivial.

(2) $\Rightarrow(1)$. For any maximal ideal $m$ of $R$, we have $\operatorname{Tor}_{n+1}(A, R / \mathfrak{m})=0$ by (2). Hence

$$
\operatorname{Tor}_{n+1}^{R_{\mathfrak{m}}}\left(A_{\mathfrak{m}}, R_{\mathfrak{m}} / \mathfrak{m}_{\mathfrak{m}}\right) \cong\left(\operatorname{Tor}_{n+1}(A, R / \mathfrak{m})\right)_{\mathfrak{m}}=0 .
$$

Since $R$ is commutative coherent, $R_{\mathrm{m}}$ is a commutative coherent local ring with only one maximal ideal $\mathrm{m}_{\mathfrak{m}}[8]$. Then $f d_{R_{\mathfrak{m}}} A_{\mathfrak{m}} \leqslant n$ by Lemma 3.2 , and hence, by Lemma 3.3 ,

$$
p d A=f d A=\sup \left\{f d_{R_{\mathfrak{m}}} A_{\mathfrak{m}} \mid m \text { is a maximal ideal of } R\right\} \leqslant n .
$$

We are now in a position to prove

THEOREM 3.5. If $R$ is a commutative coherent ring, then

(1) For any finitely presented $R$-module $A$,

$$
\begin{aligned}
p d A & =\sup \left\{n \mid \operatorname{Ext}^{n}(A, S) \neq 0 \text { for some simple } R \text {-module } S\right\} \\
& =\sup \left\{n \mid \operatorname{Tor}_{n}(A, S) \neq 0 \text { for some simple } R \text {-module } S\right\} .
\end{aligned}
$$

(In case there are no such $n$, the supremum is zero.)

(2)

$$
\begin{aligned}
w D(R) & =\sup \{f d S \mid S \text { is a simple } R \text {-module }\} \\
& =\sup \{i d S \mid S \text { is a simple } R \text {-module }\} \\
& =\sup \{F P \text {-idS } \mid S \text { is a simple } R \text {-module }\}
\end{aligned}
$$

Proof: (1) By Lemma 3.1, it suffices to prove the equality

$$
p d A=\sup \left\{n \mid \operatorname{Tor}_{n}(A, S) \neq 0 \text { for some simple } R \text {-module } S\right\} .
$$

First, we assume $p d A=m<\infty$. Then it is easily seen that

$$
\sup \left\{n \mid \operatorname{Tor}_{n}(A, S) \neq 0 \text { for some simple } R \text {-module } S\right\} \leqslant m \text {. }
$$

Since $p d A=m$, $\operatorname{Tor}_{m}(A, S) \neq 0$ for some simple $R$-module $S$ by Lemma 3.4. Then the supremum is greater than or equal to $m$, and hence the equality holds.

Secondly, suppose $p d A=\infty$. Then for any integer $n \geqslant 1$, there exists a simple $R$-module $S$ such that $\operatorname{Tor}_{n}(A, S) \neq 0$ by Lemma 3.4, and hence the supremum is greater than or equal to $n$. Thus the supremum is infinite. So we always have

$$
p d A=\sup \left\{n \mid \operatorname{Tor}_{n}(A, S) \neq 0 \text { for some simple } R \text {-module } S\right\},
$$


and the proof of $(1)$ is complete.

(2) By Lemma 3.1, it is sufficient to prove

$$
w D(R)=\sup \{f d S \mid S \text { is a simple } R \text {-module }\} .
$$

For any finitely presented $R$-module $A$, by (1),

$$
\begin{aligned}
p d A & =\sup \left\{n \mid \operatorname{Tor}_{n}(A, S) \neq 0 \text { for some simple } R \text {-module } S\right\} \\
& \leqslant \sup \{f d S \mid S \text { is a simple } R \text {-module }\} .
\end{aligned}
$$

Hence

$$
\begin{aligned}
w D(R) & =\sup \{p d A \mid A \text { is a finitely presented } R \text {-module }\} \\
& \leqslant \sup \{f d S \mid S \text { is a simple } R \text {-module }\} \leqslant w D(R)
\end{aligned}
$$

that is $w D(R)=\sup \{f d S \mid S$ is a simple $R$-module $\}$. This completes the proof.

As an immediate consequence of the Theorem 3.5 above, we have

COROLlaRY 3.6. If $R$ is a commutative Noetherian ring, then

$$
\begin{aligned}
D(R) & =\sup \{p d S \mid S \text { is a simple } R \text {-module }\} \\
& =\sup \{i d S \mid S \text { is a simple } R \text {-module }\} .
\end{aligned}
$$

(Since $R$ is commutative, we drop the unneeded letters $l$ and $r$.)

\section{Simple MOdULES OVER RIGHT SEMI-ARTINIAN RINGS}

In Section 3, it is shown that for a commutative coherent ring $R$,

$$
\begin{aligned}
w D(R) & =\sup \{f d S \mid S \text { is a simple } R \text {-module }\} \\
& =\sup \{F P \text {-idS } \mid S \text { is a simple } R \text {-module }\} .
\end{aligned}
$$

In general, the formulae fail for right coherent rings, as shown by $[7, p .348]$ and [5, Theorem 1.4, 2.3]. In this section, we prove that if $R$ is right coherent and right semi-artinian, then the above formulae hold. (In fact, the first formula holds for right semi-artinian rings.)

We start with two lemmas.

LEMMA 4.1. Let $R$ be any ring and $\mathfrak{M}$ the collection of maximal right ideals of $R$. Then the following are equivalent:

(1) Every $\mathfrak{M}$-injective right $R$-module is injective.

(2) The right $R$-module $R / E$ has non-zero socle for every proper essential right ideal $E$ of $R$. 
Proof: See Smith [14, Lemma 4].

LEmma 4.2. Let $R$ be right semi-artinian. Then

(1) For any left $R$-module $A$,

$f d A \leqslant n$ if and only if $\operatorname{Tor}_{n+1}(S, A)=0$ for all simple right $R$-modules $S$.

(2) For any right $R$-module $A$,

$i d A \leqslant n$ if and only if $\mathrm{Ext}^{n+1}(S, A)=0$ for all simple right $R$-modules $S$.

ProOF: (1) It is sufficient to prove the "if" part. We proceed by induction on $n$.

Let $n=0$. Assume $\operatorname{Tor}_{1}(S, A)=0$ for all simple right $R$-modules $S$. For any $I \in \mathfrak{M}, R / I$ is a simple right $R$-module, hence $\operatorname{Tor}_{1}(R / I, A)=0$. Let $X^{+}=\operatorname{Hom}_{z}(X, Q / Z)$ be the character module of an $R$-module $X$. Then we have an isomorphism

$$
\operatorname{Ext}^{1}\left(R / I, A^{+}\right) \cong \operatorname{Tor}_{1}(R / I, A)^{+},
$$

and hence $\operatorname{Ext}^{1}\left(R / I, A^{+}\right)=0$. Thus $A^{+}$is $\mathfrak{M}$-injective, and so $A^{+}$is injective by Lemma 4.1, that is $A$ is flat.

For $n \geqslant 1$, let

$$
\cdots \rightarrow P_{n-1} \rightarrow P_{n-2} \rightarrow \cdots \rightarrow P_{0} \rightarrow A \rightarrow 0
$$

be a projective resolution of $A$ with $K=\operatorname{Ker}\left(P_{n-1} \rightarrow P_{n-2}\right)$. Then

$$
\operatorname{Tor}_{1}(S, K) \cong \operatorname{Tor}_{n+1}(S, A)=0
$$

for all simple right $R$-modules $S$. The case $n=0$ shows $K$ is flat, whence $f d A \leqslant n$.

(2) We prove the "if" part by induction on $n$.

Let $n=0$. Then $\operatorname{Ext}^{1}(R / I, A)=0$ for all $I \in \mathfrak{M}$, and hence $A$ is $\mathfrak{M}$-injective. So $A$ is injective by Lemma 4.1 .

For $n \geqslant 1$, suppose

$$
0 \rightarrow A \rightarrow E^{0} \rightarrow \cdots \rightarrow E^{n-1} \rightarrow E^{n} \rightarrow \cdots
$$

be an injective resolution of $A$ with $L=\operatorname{Im}\left(E^{n-1} \rightarrow E^{n}\right)$. Then

$$
\operatorname{Ext}^{1}(S, L) \cong \operatorname{Ext}^{n+1}(S, A)=0
$$

for all simple $R$-modules $S$. The case $n=0$ shows $L$ is injective, and hence idA $\leqslant n$. $\square$ 
THEOREM 4.3. Let $R$ be right semi-artinian. Then

(1) $f d A=\sup \left\{n \mid \operatorname{Tor}_{n}(S, A) \neq 0\right.$ for some simple right $R$-module $\left.S\right\}$ for all left $R$-modules $A$.

(2) idA $=\sup \left\{n \mid \operatorname{Ext}^{n}(S, A) \neq 0\right.$ for some simple right $R$-module $\left.S\right\}$ for all right $R$-modules $A$.

(3) $w D(R)=\sup \{f d S \mid S$ is a simple right $R$-module $\}$.

(4) $r D(R)=\sup \{p d S \mid S$ is a simple right $R$-module $\}$.

Proof: (1) and (2) follow from Lemma 4.2.

(3) For any left $R$-module $A$, by (1),

$$
\begin{aligned}
f d A & =\sup \left\{n \mid \operatorname{Tor}_{n}(S, A) \neq 0 \text { for some simple right } R \text {-module } S\right\} \\
& \leqslant \sup \{f d S \mid S \text { is a simple right } R \text {-module }\} .
\end{aligned}
$$

Hence

$$
\begin{aligned}
w D(R) & =\sup \{f d A \mid A \text { is a left } R \text {-module }\} \\
& \leqslant \sup \{f d S \mid S \text { is simple right } R \text {-module }\} \leqslant w D(R),
\end{aligned}
$$

and (3) follows.

(4) For any right $R$-module $A$, by (2),

$$
\begin{aligned}
i d A & =\sup \left\{n \mid \operatorname{Ext}^{n}(S, A) \neq 0 \text { for some simple right } R \text {-module } S\right\} \\
& \leqslant \sup \{p d S \mid S \text { is a simple right } R \text {-module }\}
\end{aligned}
$$

whence

$$
\begin{aligned}
r D(R) & =\sup \{i d A \mid A \text { is a right } R \text {-module }\} \\
& \leqslant \sup \{p d S \mid S \text { is a simple right } R \text {-module }\} \\
& \leqslant r D(R)
\end{aligned}
$$

and so (4) holds.

We obtain the following result of [4] immediately from Theorem 4.3 above.

Corollary 4.4. If $R$ is a semi-artinian and right $S F$-ring, then $R$ is a von Neumann regular ring.

Since $R$ is left perfect if and only if $R$ is right semi-artinian and semi-local [16], we have the following result of [13] as a corollary.

Corollary 4.5. If $R$ is a left perfect ring with Jacobson radical $J$, then

$$
l D(R)=w D(R)=f d(R / J) \text { and } r D(R)=p d(R / J)
$$


where $R / J$ is considered as a right $R$-module.

Proof: Immediate since every simple right $R$-module is a direct summand of the right $R$-module $R / J$ by [9, Theorem 9.3.4].

The proof of the next main result requires a lemma.

Lemma 4.6. Let $R$ be right semi-artinian and right coherent and $A$ a finitely presented right $R$-module. Then

$p d A \leqslant n$ if and only if $\operatorname{Ext}^{n+1}(A, S)=0$ for all simple right $R$-modules $S$.

ProOF: It suffices to prove the "if" part.

"If" part. Let $B$ be any right $R$-module. We define $\left\{B_{\alpha}\right\}$ inductively. Let $B_{0}=0$, $B_{1}=\operatorname{Soc}(B)$. For any ordinal $\alpha$, if $a$ is not a limit ordinal, let $B_{\alpha}$ be a submodule of $B$ such that $B_{\alpha} / B_{\alpha-1}=\operatorname{Soc}\left(B / B_{\alpha-1}\right)$; if $\alpha$ is a limit ordinal, let $B_{\alpha}=\bigcup_{\beta<\alpha} B_{\beta}$. By the transfinite construction principle, $\left\{B_{\alpha}\right\}$ is well-defined. Since $R$ is right semiartinian, $B$ is a right semi-artinian $R$-module. Thus $B=B_{\alpha_{0}}$ for some ordinal $\alpha_{0}$ by $[16$, p.183].

Next we use transfinite induction to prove that $\operatorname{Ext}^{n+1}\left(A, B_{\alpha}\right)=0$ for all ordinals $\alpha$. In fact, if $\alpha=0$, then $B_{0}=0$. Of course, $\operatorname{Ext}^{n+1}\left(A, B_{0}\right)=0$. For each ordinal $\alpha>0$, assume $\operatorname{Ext}^{n+1}\left(A, B_{\beta}\right)=0$ for all $\beta<\alpha$. If $\alpha$ is not a limit ordinal, then we have an exact sequence

$$
0 \rightarrow B_{\alpha-1} \rightarrow B_{\alpha} \rightarrow B_{\alpha} / B_{\alpha-1} \rightarrow 0
$$

Since $B_{\alpha} / B_{\alpha-1}=\operatorname{Soc}\left(B / B_{\alpha-1}\right)$ is semisimple, $B_{\alpha} / B_{\alpha-1}=\bigoplus_{j} S_{j}$, where each $S_{j}$ is simple. Thus

$$
\operatorname{Ext}^{n+1}\left(A, B_{a} / B_{\alpha-1}\right)=\operatorname{Ext}^{n+1}\left(A, \bigoplus_{j} S_{j}\right) \cong \bigoplus_{j} \operatorname{Ext}^{n+1}\left(A, S_{j}\right)=0
$$

by $\left[15\right.$, Theorem 3.2]. But $\operatorname{Ext}^{n+1}\left(A, B_{\alpha-1}\right)=0$ by induction hypothesis, and so

$$
\operatorname{Ext}^{n+1}\left(A, B_{\alpha}\right)=0 \text {. }
$$

If $\alpha$ is a limit ordinal, then $B_{\alpha}=\bigcup_{\beta<\alpha} B_{\beta}=\underline{\lim } B_{\beta}$, and hence

$$
\operatorname{Ext}^{n+1}\left(A, B_{\alpha}\right) \cong \lim _{\longrightarrow} \operatorname{Ext}^{n+1}\left(A, B_{\beta}\right)=0
$$


again by $\left[15\right.$, Theorem 3.2]. Thus $\operatorname{Ext}^{n+1}\left(A, B_{\alpha}\right)=0$ for all $\alpha$, in particular,

$$
\operatorname{Ext}^{n+1}(A, B)=0 \quad\left(\text { for } B=B_{\alpha_{0}}\right) \text {, }
$$

whence $p d A \leqslant n$.

THEOREM 4.7. Let $R$ be right semi-artinian and right coherent. Then

(1) $p d A=\sup \left\{n \mid \operatorname{Ext}^{n}(A, S) \neq 0\right.$ for some simple right $R$-module $\left.S\right\}$ for all finitely presented right $R$-modules $A$.

(2) $w D(R)=\sup \{F P$-idS $\mid S$ is a simple right $R$-module $\}$.

Proof: (1) follows from Lemma 4.6.

(2) For any finitely presented right $R$-module $A$, by (1),

$$
\begin{aligned}
p d A & =\sup \left\{n \mid \operatorname{Ext}^{n}(A, S) \neq 0 \text { for some simple right } R \text {-module } S\right\} \\
& \leqslant \sup \{F P \text {-idS } \mid S \text { is a simple right } R \text {-module }\}
\end{aligned}
$$

Then

$$
\begin{aligned}
w D(R) & =\sup \{p d A \mid A \text { is a finitely presented right } R \text {-module }\} \\
& \leqslant \sup \{F P \text {-idS } \mid S \text { is a simple right } R \text {-module }\} \\
& \leqslant \sup \{F P \text {-idN } \mid N \text { is a right } R \text {-module }\}=w D(R)
\end{aligned}
$$

by $[15$, Theorem 3.3$]$, and so (2) follows.

\section{REFERENCES}

[1] F.W. Anderson and K.R. Fuller, Rings and categories of modules (Springer-Verlag, Berlin, Heidelberg, New York, 1973).

[2] M. Auslander, 'On the dimension of modules and algebras (III), global dimension', Nagoya Math. J. 9 (1955), 67-77.

[3] S.U. Chase, 'Direct products of modules', Trans. Amer. Math. Soc. 97 (1960), 457-473.

[4] J.L. Chen, 'On von Neumann regular rings and $S F$-rings', Math. Japon. 36 (1991), 1123-1127.

[5] J.H. Cozzens, 'Homological properties of the ring of differential polynomials', Bull. Amer. Math. Soc. 76 (1970), 75-79.

[6] D.J. Fieldhouse, 'Character modules, dimension and purity', Glasgow Math. J. 13 (1972), 144-146.

[7] K.L. Fields, 'On the global dimension of residue rings', Pacific J. Math. 32 (1970), 345-349.

[8] M.E. Harris, 'Some results on coherent rings', Proc. Amer. Math. Soc. 17 (1966), 474-479.

[9] F. Kasch, Modules and rings (Academic Press, London, New York, 1982). 
[10] B.L. Osofsky, 'Global dimension of valuation rings', Trans. Amer. Math. Soc. 126 (1967), 136-149.

[11] J. Rainwater, 'Global dimension of fully bounded Noetherian rings', Comm. Algebra 15 (1987), 2443-2456.

[12] J.J. Rotman, An introduction to homological algebra (Academic Press, New York, 1979).

[13] F.L. Sandomierski, 'Homological dimension under change of rings', Math. Z. 130 (1973), 55-65.

[14] P.F. Smith, 'Injective modules and prime ideals', Comm. Algebra 9 (1981), 989-999.

[15] B. Stenström, 'Coherent rings and FP-injective modules', J. London Math. Soc. 2 (1970), 323-329.

[16] B. Stenström, Rings of Quotients (Springer-Verlag, Berlin, Heidelberg, New York, 1975).

[17] M.L. Teply, 'Global dimensions of right coherent rings with left Krull dimension', Bull. Austral. Math. Soc. 39 (1989), 215-223.

[18] R. Ware, 'Endomorphism rings of projective modules', Trans. Amer. Math. Soc. 155 (1971), 233-256.

Department of Mathematics

Nanjing University

Nanjing 210008

Peoples Republic of China
Department of Mathematics and Mechanics

Southeast University

Nanjing 210018

Peoples Republic of China 